\title{
Development of aperture total internal reflection (A-TIR) for droplet characterization with $3-\mathrm{D}$ ray tracing and modified Fresnel equation Iltai Isaac Kim ${ }^{1 *}$, Yang Lie ${ }^{1}$, and Jaesung Park ${ }^{1}$
}

\author{
${ }^{1}$ Texas A\&M University-Corpus Christi, 6300 Ocean Dr. Corpus Christi, TX 78412-5797, USA
}

\begin{abstract}
This study proposes and demonstrates a new approach using aperture total internal reflection (A-TIR) by means of various apertures in front of a detector to characterize micro/macro droplet. Pure liquid is used to make micro and macro scale droplets. An aperture in front of a detector in TIR configuration generates unique reflectance curve by filtering the amount of aberrated beams from the top curved profiles of droplets. A scheme of threedimensional (3-D) ray tracing is developed for the reflected beam profile from the curved surface of the droplet with the modified Fresnel modeling to show a good agreement with the measurement. The modified Fresnel modeling is proposed to consider the morphological features of the droplet such as the thickness, the diameter, the surface coverage fraction, the effective flatness ratio, and the quantum phenomenon of Goos-Hänchen (G-H) shift effect. Various sizes of apertures are employed to demonstrate the A-TIR reflectance dependence on the aperture sizes for the macro and micro-sized droplets with a good agreement between the experiment and the simulation. and 3-D ray tracing with the modified Fresnel equation shows good agreement. Furthermore, it is demonstrated that one of the morphological features of the droplet, the thickness can be successfully determined with a reasonable agreement with the measurement. This outcome can be used to determine the morphological features of droplets such as the thickness, the diameter, and the coverage fraction and to characterize uneven surface features like human fingerprinting.
\end{abstract}

KEY WORDS: Aperture total internal reflection, micro/macro droplet, 3-D ray tracing, Fresnel equation, fingerprinting

\section{INTRODUCTION}

Fingerprint pattern characterization has been attracting attention in the development of antifouling coating materials in the semiconductor industry [1], the forensic analysis of aging fingerprints and chemical effects [2], and security pattern recognition [3]. In coating materials development for antifouling, there are not many substantial studies or standard protocols about the characterization of fingerprint patterns on different surface coatings. The characterization of fingerprints with real human sebum is challenging because of its complicated shape as reported in the recent publication [4] as the real human sebum contains various kinds of liquids and solid particles like a turbid medium, causing difficulty in obtaining consistent measurements. The recent study [4] shows that microscopic imaging of fingerprint patterns with pure liquids consist of numerous micro droplets and its measured reflectance with a fixed and small aperture can be explained with the modified Fresnel equation modelling in the proposed technique of aperture total internal reflectance (A-TIR) technique.

This paper is expanding the recent study to provide experimental verification for the reflected broad beam profile from the droplet using 3-D ray tracing and modified Fresnel equation modelling, study the effect of the various aperture sizes in the measured reflectance curves and its impact in the determining the droplet morphological features such as the thickness, diameter, and the surface coverage fraction, which has never been conducted before, to the authors' knowledge.

The proposed A-TIR technique can characterize various-size droplets and fingerprint patterns by placing apertures in front of a light detector and selectively blocking aberrated beams from the top curved profiles of droplets and liquid-air interfaces due to quantum shift effects. With an aperture, the measured reflectance shows a unique trend by cutting off the scattered or deviated beams compared with a classical 
measurement without an aperture. The Fresnel equation is modified with parameters to consider the geometrical features of droplet patterns and the quantum effects of the Goos-Hänchen shift [5-8]. Parameters are quantitatively estimated by analysing the geometry of droplet profiles. Micro [9] and macro size droplets are used using A-TIR technique to explain the origin of the reflected broad beam profile from the droplet, to investigate the effect of the various apertures, and to show how to determine the droplet morphological features such as the thickness and the diameter, which can provide an alternative way to measure micron-range thickness in an effective way. The existing method to measure this small thickness is only possible through the counting the number of the fringes in interference using the sophisticated microscope. A-TIR can be also coupled with the internal reflection interference technique to determine other droplet morphological features such as the contact angle, the thickness of the precursor, and the droplet profiles, which will be presented and reported separately.

\section{APERTURE TOTAL INTERNAL REFLECTION (A-TIR) THEORY}

Total internal reflection occurs when a wave propagating in a medium of higher refractive index $\left(n_{1}\right)$ strikes a boundary surface with another medium of lower refractive index $\left(n_{2}\right)$ at an angle larger than a particular critical angle $\left(\theta^{*}\right)$ with respect to the normal to the boundary [10]. The critical angle is the angle of incidence above which total internal reflection occurs, which is dependent on the refractive indices of both media on the boundary and determined by Snell's law of $n_{1} \sin \theta^{*}=n_{2} \sin \left(90^{\circ}\right)$. Total internal reflection associated with a liquid droplet on a glass substrate involves two critical angles that result from two interfaces (liquid-glass and vapor-glass).

The optical ray tracing about two critical angles are illustrated in Fig. 1. In the case of an incident angle larger than the critical angle of the liquid-glass interface, the propagated wave is totally reflected back internally at the interface ( $R f l \_1$ and $R f l \_2$ in Fig. 1-a). Meanwhile, if the incident angle is lower than the critical angle of the liquid-glass interface but higher than that of the vapor-glass interface, the wave propagation becomes more complicated. The wave striking the vapor-glass interface is totally reflected back (Rfl_1), but the wave reaching the liquid-glass interface under a droplet will be divided into two components; one is reflected at the liquid-glass interface $\left(R f l \_2\right)$ and the other is transmitted through the liquid and is reflected at the vapor-liquid interface, i.e. the upper curved boundary of the droplet $\left(R f l \_3, R f l \_b l o c k e d, T_{\text {scatt }}\right)$. The waves reflected at the curved droplet surface are not parallel to each other as in Fig. 1-b and direct to different ways depending on the incident angle normal to the profile of droplet surface. A portion of the waves striking the top of the droplet surface is internally reflected back with a similar angle $\left(R f l \_3\right)$ as the reflections at the interfaces of the vapor-glass ( $\left.R f l \_1\right)$ and liquid-glass $\left(R f l \_2\right)$ interfaces. The other waves striking the sides of the droplet surface will have severe aberration in their propagation ( $R f l \_$blocked and $T_{-}$scatt) and will not be captured in the aperture-mounted detector.

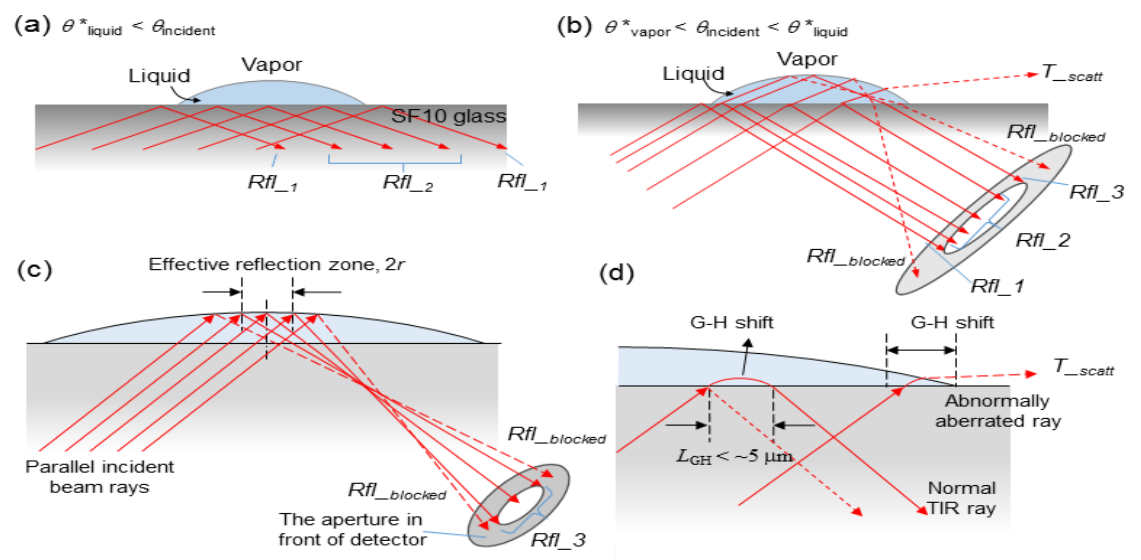

Fig. 1 Illustration of aperture total internal reflection (A-TIR) occurring with micro droplet on glass substrate. Ray tracing with two different range of incident angle (a) $\theta *{ }_{\text {liquid }}<\theta_{\text {incident }}$ and (b) $\theta^{*}{ }_{\text {vapor }}<\theta_{\text {incident }}<\theta^{*}$ liquid. (c) Concept of effective reflection zone and aperture for specific reflectance curves. (d) Effect of GoosHänchen shift on the aberration of TIR beam path [4]. 
Fig. 1-c shows the effective reflection zone at the interface of liquid and vapor where only a small portion of the waves around the droplet's apex can pass through the aperture and be captured at the detector, while other waves are blocked because of large aberration.

The other factor to consider in A-TIR measurement is the aberration of the beam path due to Goos-Hänchen effects occurring near the droplet's edge (Fig. 1-d). The Goos-Hänchen (G-H) effect is a quantum phenomenon caused by the lateral shift of reflected waves in TIR mode and the axial shift of the penetrated waves into a medium [5-8]. The lateral shift corresponds to a displacement $L_{\mathrm{GH}}$ along the interface of the incident plane (see Fig. 1-d). If the incident wave strikes close to the contact line of the droplet, the wave could come out of the vapor-liquid interface and be scattered into the vapor, not captured by the detector. The axial shift is not considered here as most samples have thickness greater than the general penetration depth of $300 \mathrm{~nm}$ [5].

Thus, the parallel-reflected waves $\left(R f l \_1, R f l \_2\right.$, and $\left.R f l \_3\right)$ can be selectively detected by placing an aperture in front of a detector as in Fig. 1-b. The total reflectance $R$ measured at the aperture-mounted detector consists of three individual reflectance paths as a function of incident angle:

$$
R=R f l \_1+R f l \_2+R f l \_3
$$

The aperture produces the unique pattern of the reflectance curve, which is a characteristic of this measurement distinguished from the classical method. The morphological features of the droplet pattern can be obtained from the measured reflectance curves. Without an aperture, the reflectance curve would be similar to those seen in classical TIR measurements.

This research is dealing with a novel use of TIR measurement whose data have never been reported previously. It is intriguing to search for the right modeling for the experimental data. In this study, the Fresnel equation is modified with parameters to simulate the reflectance measured with an aperture-mounted detector in TIR configuration. Fig. 2 shows the optical ray-tracing for the three parallel-reflected rays $\left(R f l \_1, R f l \_2\right.$ and $R f l \_3$ ) captured in the aperture-mounted detector in Fig. 1-b, where a liquid droplet is on a slide glass placed on an equilateral triangle prism in an internal reflection configuration. The incident light beam entering the prism is divided into three different paths depending on the interfaces on which the beam is reflected. Each reflectance will be expressed with the modified Fresnel equations for each ray with the corresponding parameters reflecting the geometric features of the droplet pattern. The parameters will be described in detail in the following sections.

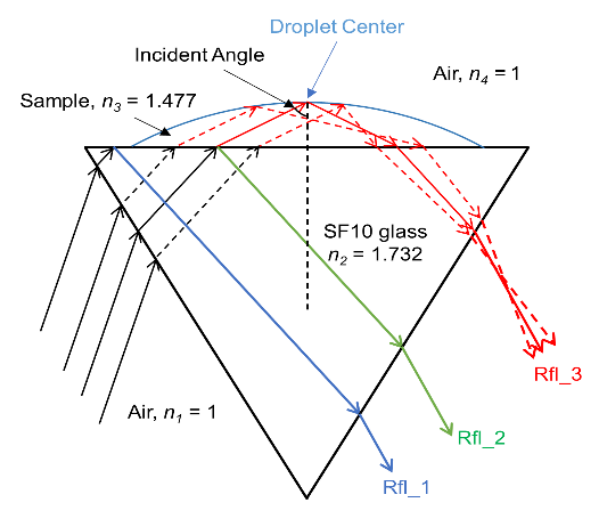

Fig. 2 Optical ray-tracing generated from the total internal reflection with a liquid droplet on a glass substrate based on an equilateral triangle prism.

\subsection{The reflectance at glass-air interface $\left(R f l \_1\right)$ and coverage fraction $(\beta)$}

The ray reaches the glass-air interface only in areas that are not covered by liquid droplets on the glass surface. The ray is totally (if $\theta_{\text {incident }} \geq 35.3^{\circ}$ ) or partially (if $\theta_{\text {incident }}<35.3^{\circ}$ ) reflected depending on the incident angle ( $\left.\theta_{\text {incident }}\right)$, which is indicated as "Rfl_1" in Fig. 2. The modified reflectance of this ray is based on Fresnel equations with some parameters as follows:

$$
R f l \_1=\alpha(1-\beta)\left(1-\gamma_{1}\right)\left|t_{12}\right|^{2}\left|r_{21}\right|^{2}\left|t_{21}\right|^{2}
$$

where $t$ and $r$ are the transmission and reflection coefficients of the Fresnel equation, the subscripts ' 1 ' and ' 2 ' indicate the medium of air and glass, respectively, $\alpha$ is the transmission ratio, and $\beta$ is the coverage fraction. The transmission and reflection coefficient are as follows: 


$$
\begin{aligned}
t_{p} & =\frac{2 n_{i} \cos \theta_{i}}{n_{t} \cos \theta_{i}+n_{i} \cos \theta_{t}} \\
t_{s} & =\frac{2 n_{i} \cos \theta_{i}}{n_{i} \cos \theta_{i}+n_{t} \cos \theta_{t}} \\
r_{p} & =\frac{n_{t} \cos \theta_{i}-n_{i} \cos \theta_{t}}{n_{t} \cos \theta_{i}+n_{i} \cos \theta_{t}} \\
r_{s} & =\frac{n_{i} \cos \theta_{i}-n_{t} \cos \theta_{t}}{n_{i} \cos \theta_{i}+n_{t} \cos \theta_{t}}
\end{aligned}
$$

The subscripts ' $\mathrm{i}$ ' and ' $\mathrm{t}$ ' indicate the incident and transmitted media at interface, respectively. $\left|t_{12}\right|^{2}$ and $\left|t_{21}\right|^{2}$ are the transmittances occurring at both side interfaces of the prism where the wave enters and exits, respectively. $\left|r_{21}\right|^{2}$ is the reflectance of the wave propagating from glass to air on the top of prism, and it has a maximum of 1 at an incident angle larger than $35.3^{\circ}$ normal to the air-glass boundary. The last parameter of $\gamma 1$ is related to the additional attenuation of reflectance $\left|r_{21}\right|^{2}$ particularly near the critical angle of $35.3^{\circ}$, which is caused by Goos-Hänchen (G-H) shift which will be explained in the following section 2.2. This parameter will be described in detail with the discussion of the G-H effect in the following section.

The first parameter $\alpha$ is the transmission ratio through the substrate medium composed of a prism, a slide glass, and an index-matching fluid. In this experiment, $\alpha$ was empirically estimated as approximately $95 \%$ by comparing the intensities of the entering and exiting beams. Experimental measured reflectance of $R f l \_1$ agrees well with the calculation by Eq. 2 with $\alpha=95 \%$. This $95 \%$ value of $\alpha$ is consistently applied to all equations in this paper.

The second parameter $\beta$ is the coverage fraction of droplets on the reflection zone of the glass surface. It is determined as the ratio of the measured area of droplets $\left(A_{d r p}\right)$ within the reflection zone and total area of the reflection zone $\left(A_{\text {rfl }}\right)$ :

$$
\beta=\frac{A_{\text {drplets }}}{A_{r f l}}
$$

The area of the droplets, $A_{\text {drplets }}$ is determined from the image processing of the droplet pattern using Matlab software. The area of the reflection zone, $A_{r f l}$ is based on the plane-cylinder intersection shaping an ellipse that has an enlarged reflection length with increasing incident angle (Fig. 3) and can be calculated as $\pi b w$ where $b$ is the reflection length and $w$ is the laser width. The area of the droplets is determined numerically through the image processing algorithm. The coverage fraction $\beta$ slightly changes depending on the incident angles and the surface coating conditions. The change in coverage fraction $\beta$ due to varying incident angles is less than $1 \%$ for the most droplet radius as in Fig. 3, small but substantial variation the simulated reflectance curves. Eq. 2 is formulated with the uncovered area portion, $(1-\beta)$ since $R f l \_1$ is about the reflectance at the air-glass interface of $\left|r_{21}\right|^{2}$.
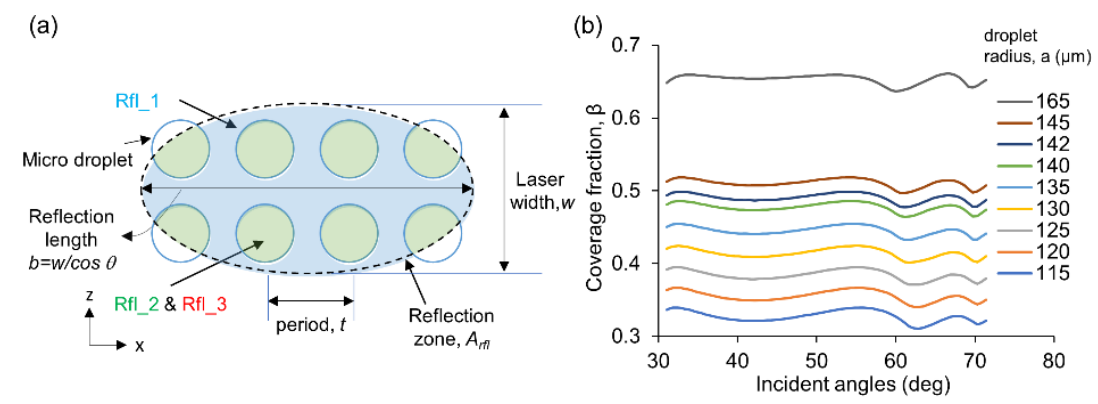

Fig. 3 Coverage fraction of $\beta$ as a function of incident angles for various droplets. $a$ is the radius of the droplet.

\subsection{The reflectance at the glass-liquid interface $\left(R f l \_2\right)$ and the Goos-Hänchen shift ratio ( $\left.\gamma\right)$} The ray strikes a glass-liquid interface under a droplet, called " $R f l \_2$ " in Fig. 3. It can be reflected totally (if $\theta_{\text {incident }} \geq 58.5^{\circ}$ ) or partially (if $\theta_{\text {incident }}<58.5^{\circ}$ ) depending on the incident angles. The modified reflectance of the second path is expressed as:

$$
R f l \_2=\alpha \beta\left(1-\gamma_{2}\right)\left|t_{12}\right|^{2}\left|r_{23}\right|^{2}\left|t_{21}\right|^{2}
$$


where $\left|r_{23}\right|^{2}$ is the reflectance of the wave at the interface between a slide glass and a droplet. The subscript ' 3 ' indicates the sample medium. Since $R f l \_2$ is the reflectance for the covered area of droplets on glass surface, the coverage fraction $\beta$ is used - unlike the reflectance of the first ray, $R f l \_1$.

The parameter $\gamma_{2}$ is the attenuation factor of the reflectance of the second ray, $R f l \_2$, similar to $\gamma_{1}$ in the reflectance of the first ray, $R f l \_$. In general, $\left|r_{23}\right|^{2}$ is maximized at an incident angle larger than the critical TIR angle of the glass-liquid interface. However, $\left|r_{23}\right|^{2}$ can be attenuated above the critical angle of $58.5^{\circ}$ when the Goos-Hänchen (G-H) shift affects the beam path. The G-H effect is a quantum phenomenon in which a polarized beam undergoes a minute lateral shift along the interface of the incident plane under TIR mode [58]. The lateral G-H shift $\left(L_{\mathrm{GH}}\right)$ for $p$ - and $s$-polarization can be expressed as:

$$
\begin{aligned}
& \left(L_{G H}\right)_{p}=\frac{1}{\pi \cos \theta} \cdot \frac{K \sin \theta \cos ^{2} \theta}{K^{2} \cos ^{2} \theta+\sin ^{2} \theta-n^{2}} \cdot \frac{\lambda_{1}}{\left(\sin ^{2} \theta-n^{2}\right)^{\frac{1}{2}}} \\
& \left(L_{G H}\right)_{S}=\frac{1}{\pi \cos \theta} \cdot \frac{\mu \sin \theta \cos ^{2} \theta}{\mu^{2} \cos ^{2} \theta+\sin ^{2} \theta-n^{2}} \cdot \frac{\lambda_{1}}{\left(\sin ^{2} \theta-n^{2}\right)^{\frac{1}{2}}}
\end{aligned}
$$

where $n^{2}=K \mu$, and for glasses $n^{2}=K$. Also, $n=n_{2} / n_{1}$ and $\lambda_{1}=\lambda_{\text {vacuum }} / n_{1}$. The lateral G-H shift is calculated as around a few microns at the critical angles of the air-glass and liquid-glass interfaces and exponentially decreases away from it. The conceptual idea of the G-H effect on the attenuation of reflectance in droplet patterns is explained in detail in the recent study [4].

\subsection{The reflectance at the liquid-air interface (Rfl_3)}

The ray passes through the glass-liquid interface with an incident angle lower than the critical angle of $58.5^{\circ}$, which is shown as " $R f l \_3$ " in Fig. 2. To be precise, this path is derivative from the partial reflection of the second ray with an incident angle from $35.3^{\circ}$ to $58.5^{\circ}$ normal to the glass-liquid interface. It is total-internally reflected at the upper boundary of the droplet, i.e. the liquid-air interface, and then turns back to the liquidglass interface at the bottom of the droplet with an external reflection mode. A part of light passes through the liquid-glass interface and finally exits the prism.

\section{3-D ray tracing with the modified Fresnel equation}

Three-dimensional (3-D) ray tracing is employed in order to calculate the Rfl_3 exactly, the with the modified Fresnel equation. For 3-D ray tracing, the laser intensity is experimentally measured as in Fig. 4 with two-Gaussian functions.
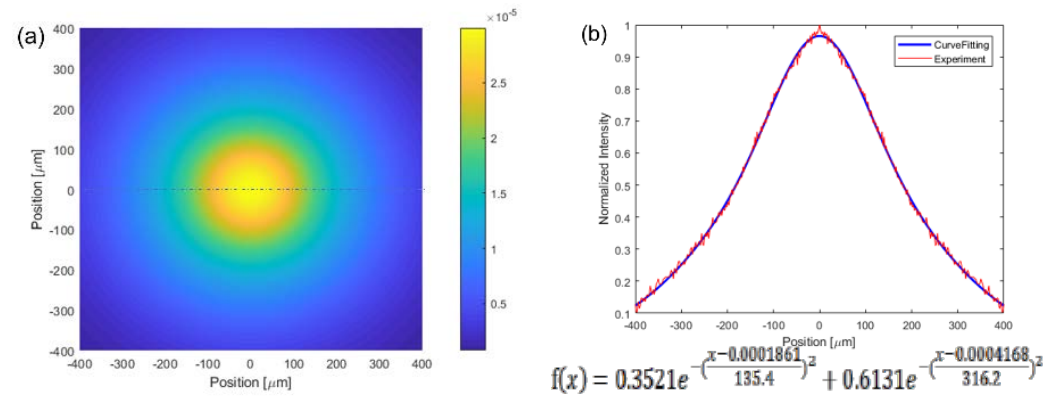

Fig. 4 The experimentally measured laser intensity distribution (a) and the centerline distribution along the center dash-dot line (b) with its curve fitted line.

With this measured intensity distribution, $R f l \_3$ is calculated using the following expression;

$R f l_{-} 3=\sum \frac{I_{i, x z}}{I_{\text {incoming }}}=\sum_{z} \sum_{x} \alpha \beta^{\prime}\left|t_{12, x z}\right|^{2}\left|t_{23, x z}\right|^{2}\left|r_{31, x z}\right|^{2}\left|t_{32, x z}\right|^{2}\left|t_{21, x z}\right|^{2}$

where $\beta^{\prime}=\frac{\kappa_{x z}}{I_{\text {incoming }}}$ and $\kappa_{x z}$ is the curve fitted function for the measured laser intensity as in Fig. 4 and the summation of $\kappa_{, x z}$ across the laser cross-sectional area is equal to the incoming laser intensity, $I_{\text {incoming. }}$.

Fig. 5 illustrates the schematic of the 3-D ray tracing calculation. The top view in the figure shows the lens effect resulting in the accumulation of the ray reflected on the top curved liquid-air interface in the offaxis locations. The laser intensity is divided with an $1 \mu \mathrm{m}$ interval for its $800 \mu \mathrm{m}$ diameter. Ray tracing is conducted for each ray. Fresnel reflection and transmission calculation are done with the ray tracing. 3-D ray tracing requires much more calculation and algorithm than the initially tried 2-D calculation. The program is developed with Matlab software and the calculated node is $\sim 6$ million elements with the horizontal (x-axis), 
the depth (z-axis), and the vertical (y-axis) directions with the different incidence angles from 30 to 70 degrees with around 1 degree interval. The zoom-in view of the overview shows the schematic of the ray tracing on the screen with $R f l \_1, R f l \_2$ and $R f l \_3$ with blue, green, and red color, respectively, with $R f l \_1$ and $R f l \_2$ targeting around the center region as they are reflections of laser light on the plane interface between the glassair and glass-liquid, respectively. $R f l \_3$ presents the dispersed beam profile in vertically (mostly in y-axis) and in depth direction (in z-axis). The spherical shape of droplet causes the lens effect and its refracted rays deviate significantly in vertical direction (in y-axis). Hence, the apertures with various sizes from 2 to $10.3 \mathrm{~mm}$ in diameter, placed in front of the detector with the size of $10.3 \mathrm{~mm}$ in diameter, are expected to collect the signatures of the droplets profile such as the thickness and the diameter. The simulation code models all geometrical considerations and Fresnel equation with the three main loops; the depth direction (z-axis), the horizontal direction (x-axis), and the incidence angles from 30 to 70 degrees.

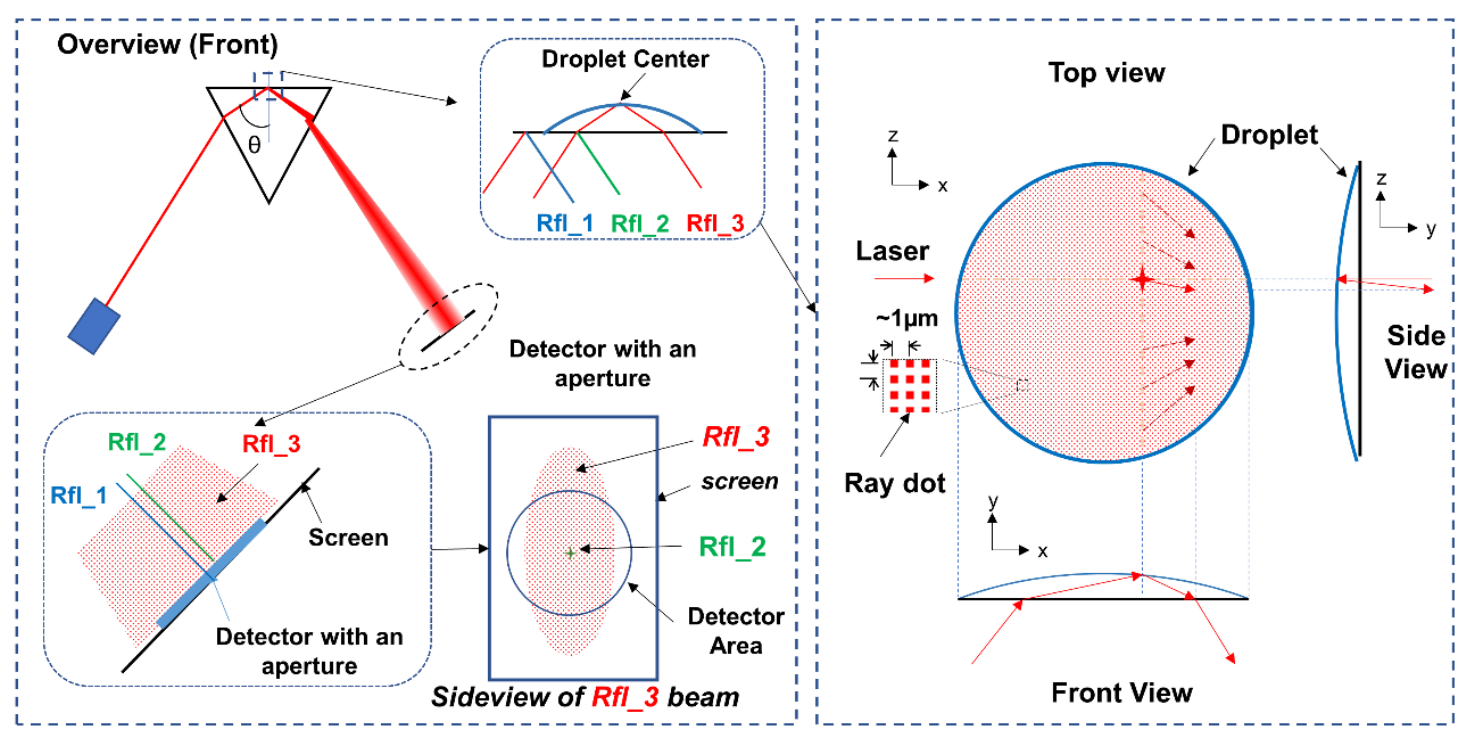

Fig. 5 A schematic of 3-D ray tracing with the modified Fresnel equation.

\section{2-D modeling with the flatness effect $(\delta)$}

Furthermore, 2-D simple modeling is conducted for the $R f l \_3$ calculation. $R f l \_3$ can be calculated as the following simple expression with the parameter of the flatness efficiency, $\delta$;

$$
R f l \_3=\alpha \beta \delta\left|t_{12}\right|^{2}\left|t_{23}\right|^{2}\left|r_{31}\right|^{2}\left|t_{32}\right|^{2}\left|t_{21}\right|^{2}
$$

where $\delta$ is the flatness efficiency that is associated with the fraction of a droplet's upper surface that makes the third ray apparently parallel to the first and second rays. A droplet may have an effective reflection zone around its apex where the reflected ray can pass through an aperture and reach to a detector. Fig. 7 shows a geometric illustration calculating the effective reflection zone based on a spherical cap droplet profile. It also presents some relevant equations with an aperture radius of $r_{a}=1 \mathrm{~mm}$ and the distance between the sample to the detector $L=100 \mathrm{~mm}$, including the flatness effect ratio of $\delta$ that is defined as the ratio of effective reflection zone to droplet area.

$$
\delta=\frac{A_{e f f}}{A_{d r p}}=\frac{r^{2}}{a^{2}}=\frac{\left(a^{2}+h^{2}\right)^{2} \cdot r_{a}^{2}}{16 a^{2} h^{2} L^{2}}
$$

where $r$ is the radius of the effective reflection zone, $a$ is the radius of the droplet, and $h$ is the thickness of the droplet. $\delta$ ranges approximately from 0.000032 to 0.0035 depending on the difference of droplet profile due to the different size droplets. The curvature of droplet profile caused arithmetic deduction on the reflectance $\left|r_{31}\right|^{2}$. The rays scattered by the curved surface of the droplet are excluded from the aperture-mounted detector as they are seriously out of the optical axis along the direction of ray propagation. As the radius of curvature increases, the flatness ratio $\delta$ increases as well. For instance, $\delta=0.1$ is obtained in a 


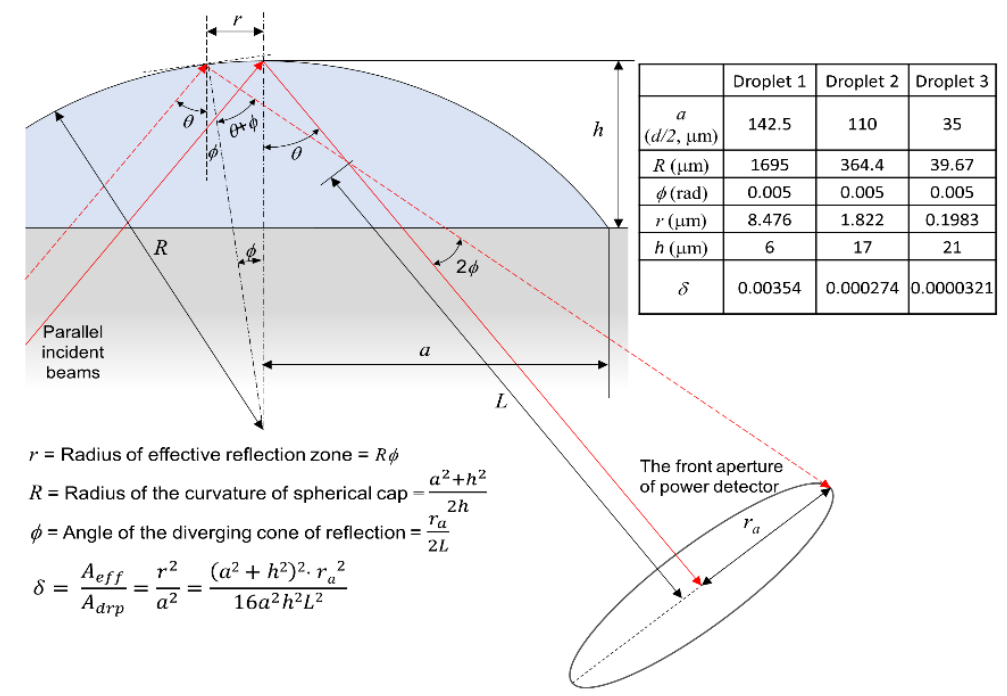

Fig. 6 2-D modeling for $R f l \_3$ calculation using the effective reflection zone of droplet surface and the relevant equations to calculate the flatness ratio, $\delta . L$ is the distance from the droplet to the detector and measured as $100 \mathrm{~mm} . r_{a}$ is the radius of the aperture, $1 \mathrm{~mm}[4]$.

droplet with a curvature radius of $16 \mathrm{~mm}$, which would be an ultra-thin droplet with a $500-\mu \mathrm{m}$ diameter and a $2-\mu \mathrm{m}$ height. The higher value of $\delta$ means more reflectance to be captured by a detector and helps TIR curves reflectance increase. The more flatness, the higher $\delta$. In addition, the effective reflection zone can increase with a larger aperture. The dependency of aperture size on the flatness ratio will be reported in the future.

The intensities of $R f l \_1, R f l \_2$, and $R f l \_3$ varies depending on the incident angles and the theoretical prediction of the reflectance curves depending on the parameters of the transmission ratio, $\alpha$, the coverage fraction, $\beta$, the $\mathrm{G}-\mathrm{H}$ shift, $\gamma$, and the flatness ratio, $\delta$ are shown in detail the recent study [4].

\section{TOTAL INTERNAL REFLECTION MEASUREMENT}

The experimental setup is illustrated in Fig. 7 with a schematic illustration on the left and a photo of the set-up on the right. An equilateral triangle prism (SF10, $n=1.732$ at $633 \mathrm{~nm}$ ) is mounted on a translation stage combined with a vertical post, and a microcontact-printed slide glass (SF10) is placed on the upper surface of the prism. An immersion oil $(n=1.730)$ is used to ensure the index matching without an air gap between the prism and the slide glass. A He-Ne laser $(\lambda=633 \mathrm{~nm})$ is mounted on a straight rail that pivots around the prism, and a digital protractor (resolution $=0.01^{\circ}$, accuracy $\left.=0.05^{\circ}\right)$ is attached to the rail. A collimated beam $(d=0.8 \mathrm{~mm})$ emitted from the laser is incident and refracted on the left surface of the prism, and then the beam passing through the prism is incident on the upper surface of the slide glass. The final incident angle on the slide glass is calculated with the rail angle measured by the protractor and Snell's law, $\sin \theta_{\text {rail }}=n_{\text {SF10 }} \sin \theta_{\text {incident }}$. The laser beam reflected internally at the glass surface comes out of the prism, and its intensity is measured by using an optical power detector while the incident angle changes from $30^{\circ}$ to $75^{\circ}$. A small aperture $\left(r_{a}=1 \mathrm{~mm}\right)$ is placed in front of the power detector in order to cut off the highly aberrated beams caused by the curved surface of the micro droplets. The reflectance is determined as the ratio of the power measured behind the cut-off aperture to the original power measured prior to entering the prism. A CCD camera is used to observe the reflected light at the same position on the glass surface by adjusting the translation stage. The triolein oil is used for the measurement with very large droplet on the slide and measured to have the refractive index of 1.477 in the recent study [4]. The microcontact printing $(\mu \mathrm{CP})$ is used to make the micro droplets on the slide [4, 12, and 13]. 

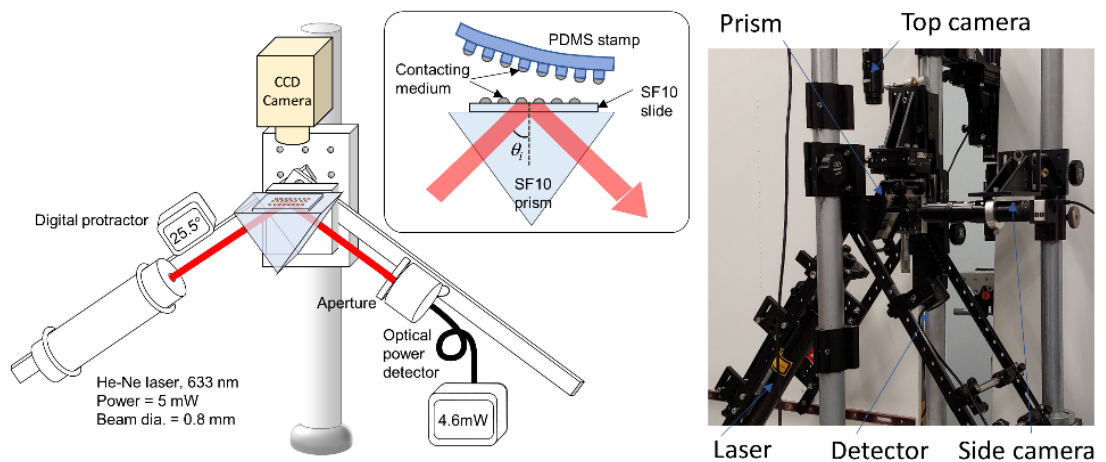

Fig. 7 Schematic diagram (left) and photograph (right) of experimental setup [4].

\section{RESULTS}

\subsection{The effect of the coverage fraction}

Aperture-TIR measurement was done for the PDMS-stamped micro droplets using an aperture-mounted detector and the measured reflectance was compared with the calculation using the modified Fresnel equation in section 3 in order to confirm the validity of the estimated parameters. Fig. 9 presents the measured reflectance from micro droplets printed by PDMS stamping on three different coating surfaces, i.e., oleophilic coating (droplet 1 in Fig. 8-a), plain glass (droplet 2 in Fig. 8-b), and oleophobic coating (droplet 3 in Fig. 8c). The presented data are the mean values of three individual measurements. The calculated reflectance is from Eqs. 1, 2, 5, and 7 with four parameters $(\alpha, \beta, \gamma$, and $\delta$ ). The value of $\alpha$ is identically 0.95 for all substrates since it is not affected by the condition of the coating surface. The other parameters $(\beta, \gamma$, and $\delta)$ are estimated differently due to the geometric features of droplet patterns according to section 2 modeling, whose numerical values are presented in each graph. Reflectance shows a decreasing trend in the middle angle range $\left(35.3^{\circ}<\right.$ $\theta_{\text {incident }}<58.5^{\circ}$ ) in the order of droplet 3,2 , and 1 with decreasing oleophobicities as the coverage fraction $\beta$ increases. Reflectance curves don't show very curved profiles around the critical angles because of the low G$\mathrm{H}$ ratio $\gamma$ (mostly less than 1\%). The calculation with the estimated parameters agrees well with the measurement, meaning the parameters are reasonably estimated.
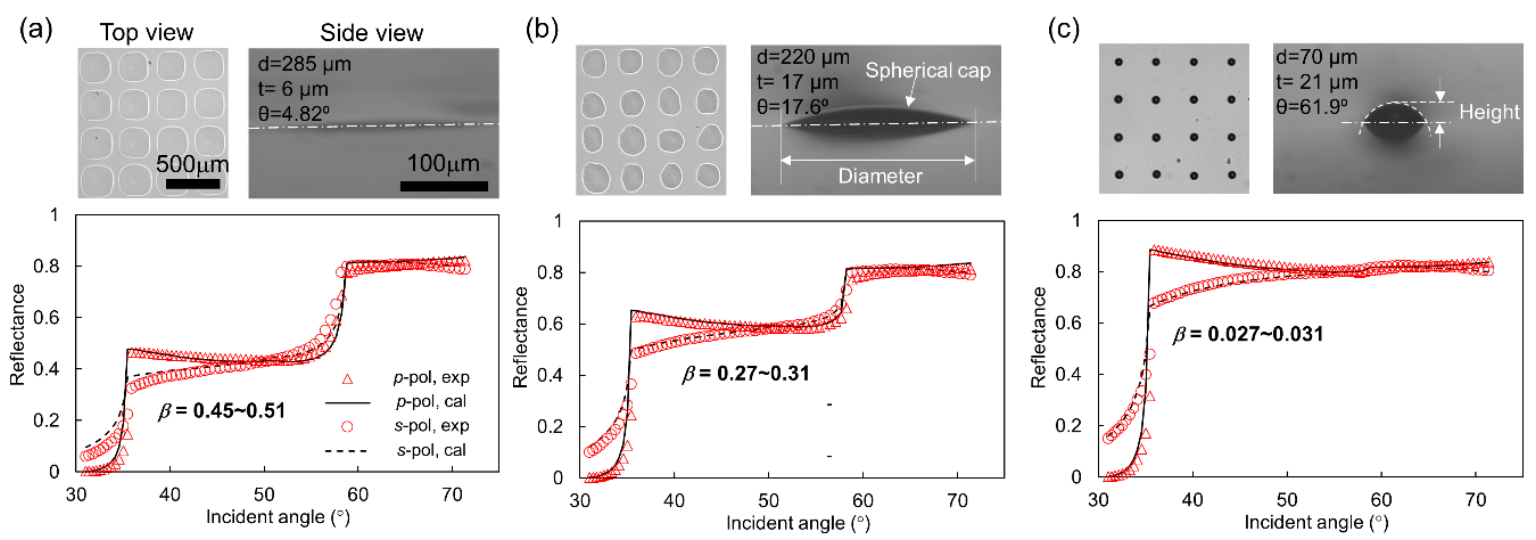

Fig. 8 The different droplet patterns and morphological parameters depending on differently coated surfaces. Reflectance curve for PDMS stamped micro-droplets on differently coated surfaces. Measurement and simulation are denoted in symbols and lines, respectively: droplet 1 on the oleophilic coating (a), droplet 2 on the plain glass (b), and droplet 3 on the oleophobic coating (c) [4].

\subsection{The reflection at the curved droplet surface, $R f l \_3$}

The reflectance at the curved surface of the droplet causes the lens effect which make the reflected beam will be beam profile through 3-D ray tracing with the modified Fresnel modeling. Macro-size droplet is used as in Fig. 10 -a as it can produce the reflected beam profiles big enough to be quickly detected. The images at top row in Fig. 10-b show the experimentally recorded Rfl_3 beam profiles on the screen using color camera at the representative 
incident angles; 54.2, 45.9, and 33.7 degrees, respectively. The images are recorded at the location of $150 \mathrm{~mm}$ away from the sample location on the prism, which gets larger with the decreasing incident angles as the optical path through the prism gets longer, resulting in more dispersion especially in vertical direction. The simulated images at the bottom row presents a good agreement with the experiment in its beam dimension and its intensity. The intensities are normalized against the maximum at the critical angle. Fig. 10-c shows the centerline normalized intensity distribution for the incidence angles in Fig. 10-b along the center dash-dot line. The experiment data are in solid symbols and the simulation results are solid lines in red, green, and blue colors for 54.2, 45.9, and 33.7 degrees, respectively, which shows a good agreement, indicating our 3-D ray-tracing with the modified Fresnel modeling works well.
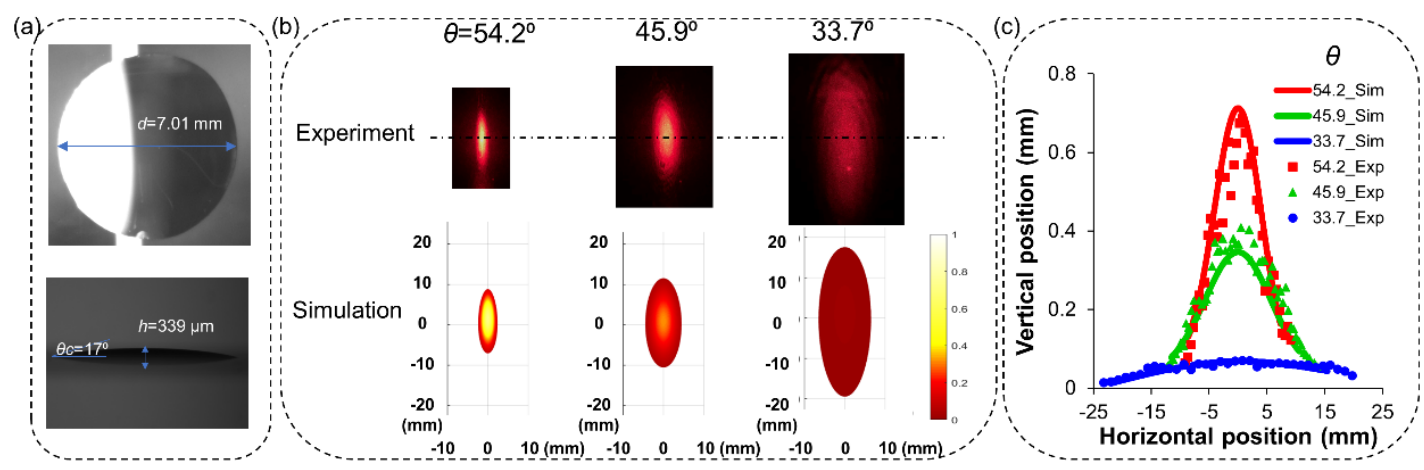

Fig. 9 Photos of macro droplet in top and side view (a), the beam profiles of $R f l \_3$ at various angles from the experiment and the simulation (b), and the centerline beam profile ( $\left.R f l \_3\right)$ (c). In $R f l \_3$ beam profile, the experiment is from the captured image on the screen in Fig. 6 and the simulation is from the 3-D ray tracing with the modified Fresnel equation. The centerline profile is along the dash-dot line in (b).

\subsection{The effect of the aperture size}

Various sized apertures are tested to explore its effect on the A-TIR reflectance in $p$ and $s$ polarization as in Fig. 10. The inset photos show the profile of its macro-size droplet; the diameter of $6.5 \mathrm{~mm}$ and the thickness of 343 $\mu \mathrm{m}$. The droplet size is big enough so that the laser is confined by the liquid droplet with targeting at the apex of the droplet. Thus, only Rfl_2 and Rfl_3 components are generated in Eq. 1 . The reflectance measured at the apertures of 2, 4, and $8 \mathrm{~mm}$ are colored in blue, red, and orange, respectively. The $p$ and $s$ polarization are shown as filled and hollow symbols for the experiment and solid and dash lines for the simulation. It shows a very good agreement between the experiment and the simulation, indicating our modified Fresnel modeling based on 3-D ray tracing describes very well the optics phenomena in aperture total internal reflection (A-TIR).

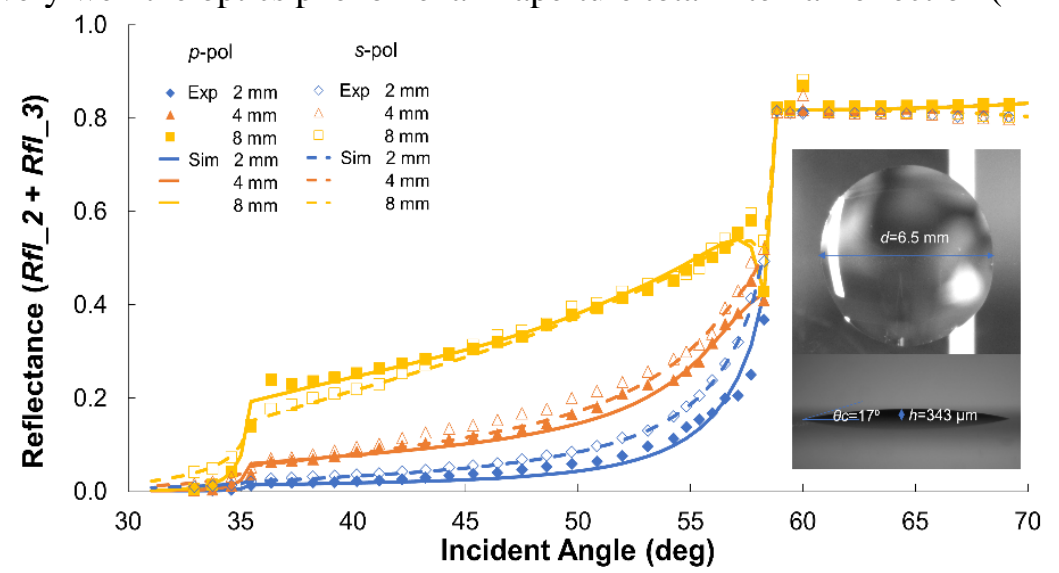

Fig. 10 Reflectance curve in $p$ and $s$ polarizations with various aperture sizes (2, 4, and $8 \mathrm{~mm}$ in diameter) versus incident angles for the macro-sized droplet measured at the location of $150 \mathrm{~mm}$ away from the sample. The inset figure shows the top and side view of the macro droplet.

After verifying the reflected beam profile ( $\left.R f l \_3\right)$ from the top curved surface and the aperture effect from the macro-sized droplet, the modified Fresnel modeling is applied to the micro droplet pattern as in Fig. 11. The left (Fig. 11-a) shows a good agreement between the experiment and the simulation for various apertures. The 
inset photo is a micro droplet pattern used. The right (Fig. 11-b) presents the contribution of each reflectance, $R f l \_1$, $R f l \_2$, and $R f l \_3$ in green, blue, and red colors, respectively, where the aperture of $10.3 \mathrm{~mm}$ contributes the maximum out of the given apertures with the aperture of $2 \mathrm{~mm}$ contributing the minimum. The variation of Rfl_3 in the right figure explains the change of the total reflectance curve in the left.
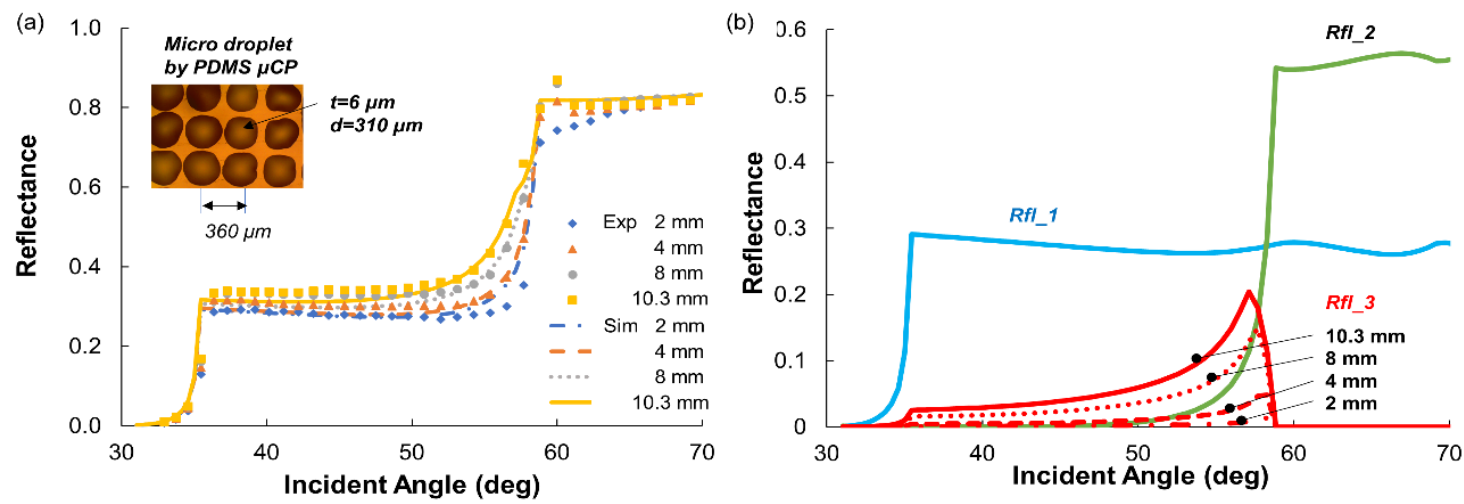

Fig. 11 Reflectance curve in p-polarization with various apertures (2, 4, 8, and $10.3 \mathrm{~mm}$ in diameter) for micro droplet pattern. (a) The total reflectance shows the experiment in symbols and the simulation in lines. (b) The contribution of each reflectance is colored as blue (Rfl_1), green ( $\left.R f l \_2\right)$, and red (Rfl_3).

\subsection{The determination of the droplet thickness}

The proposed modified Fresnel modeling can be applied to determine the morphological features of droplet such as the thickness and the diameter. The flatness ratio, $\delta$ is obtained by equaling the reflectance, $R f l \_3$ (Eq. 7) from the 3-D ray tracing with the modified Fresnel modeling to the reflectance (Eq. 8) from 2-D modeling with the flatness effect. From the determined flatness ratio, $\delta$ and the Eq. 9, the thickness can be determined as in Fig. 12 which shows a reasonable agreement with the measured thickness from Fizeau interference [14].

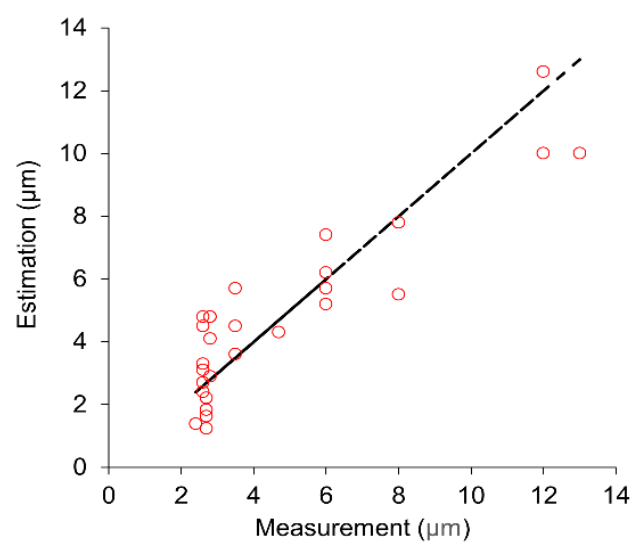

Fig. 12 Determining the thickness of the droplet

\section{CONCLUSIONS}

In this study, we completed the recently developed A-TIR technique further by experimentally verifying the origin of the reflected beam profile from the curved surface of the droplet with the modified Fresnel modeling and by demonstrating the effect of the various apertures. Furthermore, it is demonstrated that small thickness of the droplet in the range of micron can be effectively determined by A-TIR measurement, not referring to the interference method. This outcome provides new alternative method to determine the morphological features of the droplet and characterize the fingerprint patterns.

ACKNOWLEDGMENT: This study was supported by a research grant from CEKO Co., LTD and a Texas Comprehensive Research Fund (TCRF) grant from the Texas A\&M University-Corpus Christi Division of Research, Commercialization and Outreach. 


\section{REFERENCES}

[1] Zhang Z, Chao T, hen S, Jiang S. Superlow Fouling Sulfobetaine and Carboxybetaine Polymers on Glass Slides. Langmuir 2006; 22: 10072-10077, https://doi.org/10.1021/la062175d.

[2] Cadd S, Islam M, Manson P, Bleay S. Fingerprint composition and aging: A literature review. Science \& Justice 55; 2015: 219238, https://doi.org/10.1016/j.scijus.2015.02.004.

[3] Pavlidis T. A review of algorithms for shape analysis. Computer Graphics and Image Processing 1978; 7: 243-258, https://doi.org/10.1016/0146-664X(78)90115-6.

[4] J. S. Park, Y. Lie, H. J. Kim, H. C. Kim, and I. Kim, Development of aperture total internal reflection (A-TIR) for micro droplet a nd fingerprinting patterns characterization, Optics Communications, 453, 124414, 2019.

[5] Sun J, Wang, J, Liu Y, Ye Q, Zeng H, Zhou W, Mei J, Zhang C, Tian J. Effect of the gradient of complex refractive index at boundary of turbid media on total internal reflection. Opt. Express 2015; 23: 7320-7332, https://doi.org/10.1364/OE.23.007320.

[6] Renard, RH. Total Reflection: A New Evaluation of the Goos-Hänchen Shift. J. Opt. Soc. Am. 1964; 54: 1190-1197, https://doi.org/10.1364/JOSA.54.001190.

[7] Snyder AW, Love JD. Goos-Hänchen shift. Appl. Opt. 1976; 15: 236-238, https://doi.org/10.1364/AO.15.000236.

[8] Bocharov, A.A. Goos-Hänchen shift of a transmitted light beam in frustrated total internal reflection for moderately large gap widths. Optics Communications 2017; 389: 297-302, https://doi.org/10.1016/j.optcom.2016.12.047.

[9] Chou W-L, Lee P-Y, Yang C-L, Huang W-Y, Lin Y-S. Recent Advances in Applications of Droplet Microfluidics. Micromachines 2015; 6: 1249-1271, https://doi.org/10.3390/mi6091249.

[10] Nieto-Vesperinas M, Sánchez-Gil JA. Light scattering from a random rough interface with total internal reflection. J. Opt. Soc. Am. A 1992; 9: 424-436, https://doi.org/10.1364/JOSAA.9.000424.

[11] Wertz PW. Human synthetic sebum formulation and stability under conditions of use and storage. International Journal of Cosmetic Science 2009; 31: 21-25, https://doi.org/10.1111/j.1468-2494.2008.00468.x.

[12] Ruiz SA, Chen CS. Microcontact printing: A tool to pattern. Soft Matter 2007; 3: 168-177, https://doi.org/10.1039/B613349E.

[13] Perl A,Reinhoudt DN, Huskens J. Microcontact Printing: Limitations and Achievements. Advanced Materials 2009; 21: 22572268, https://doi.org/10.1002/adma.200801864.

[14] Iltai Isaac Kim, Sokwon Paik, Yangbae Jun, H.C. Kim, and H. J. Kim, Full-field thickness measurement of ultrathin liquid film in receding contact-induced nano-channel using surface plasmon resonance, Optics Express (IF: 3.356) 26, 20975, 2018. 